\title{
Inconsistency in spatial knowledge
}

\author{
IAN MOAR \\ Bucknell University, Lewisburg, Pennsylvania 17837 \\ and \\ GORDON H. BOWER \\ Stanford University, Stanford, California 94305
}

\begin{abstract}
Two experiments examined whether spatial information judged from cognitive maps contains the functional euclidean properties of real maps. In Experiment 1, the six directions between sets of three triad locations in a town were judged from memory. The angle formed by the two judged directions from a location to the other two locations in a triad was derived. The three derived angles of a triad were then summed. The derived angles were found to be biased toward 90 deg. The sum of the three derived angles of a triad aleo exceeded 180 deg, which violates the euclidean properties of real maps. In Experiment 2, subjects judged the direction between pairs of American cities in both directions. The judged directions were found to be consistently nonreversible, which is contrary to the properties of euclidean geometry. The study suggests that information judged from cognitive mapo can contain internally inconoistent spatial properties, and the results are discussed in terms of current theories of spatial representation.
\end{abstract}

Does our spatial memory for the world contain inconsistent spatial properties? Several recent studies have proposed that large-scale space is encoded using simple spatial heuristics (Byrne, 1979; Hintzman, O'Dell, \& Arndt, 1981; Stevens \& Coupe, 1978; Tversky, 1981; Wilton, 1979). For example, Byme (1979) found that subjects gave longer distance estimates for routes with many turns than for equally long routes with fewer turns. He also found a bias to remember the angle of turns as a right angle. Byrne suggested that distance along a route is estimated using the processing heuristic that the more remembered locations there are along a route between two points, the greater is the distance between those two points. Similarly, a storage heuristic is used to encode all turns as right angles. Byrne proposed a network representation for cognitive maps in which only topological kinds of information are encoded (e.g., routes as ordered strings of locations with only right-angle turns). A similar network model for route representation has been put forward by Moar and Carleton (1982).

In contrast, Stevens and Coupe (1978) have suggested a propositional hierarchical model of spatial geographic

The first experiment was carried out while the first author was at the MRC Applied Psychology Unit at Cambridge, England, and the second experiment was performed while he was a NATO postdoctoral fellow at Stanford University, California. Thanks are due to Andrea Halpern and the reviewers for helpful comments and to Beth Tanke for providing the subjects for the second experiment. Reprint requests should be sent to Ian Moar, Psychology Department, Bucknell University, Lewisburg, Pennsylvania 17837. knowledge. For example, the United States might be a superordinate in the hierarchy, with California as one of its subordinates. California, in turn, might be a superordinate to cities such as San Diego. Spatial information about a location is stored in the hierarchy using simple storage heuristics. A location is encoded in terms of its spatial relation to its immediate superordinate and its immediate subordinates. For example, California might be stored in terms of its spatial relation to its superordinate (e.g., on the southwestern coast of the United States) and its subordinates (e.g., San Diego at the southwestern corner of the state). A location can also be encoded in terms of its spatial relation to other locations at the same level in the hierarchy that share the same immediate superordinate. For instance, the spatial relation between San Diego and Los Angeles might be encoded if they share California as their immediate superordinate. These simple storage heuristics reduce the number of spatial relations that are stored and minimize storage redundancy. Spatial relations not explicitly stored have to be inferred from the stored information. For instance, the spatial relation between San Diego, California, and Reno, Nevada, may not be explicitly stored but can be inferred from the explicitly stored relations between San Diego and California, California and Nevada, and Nevada and Reno. However, distortions can occur using these inferences. If California is stored as being west of Nevada, then San Diego might be falsely inferred to be west rather than east of Reno. When Stevens and Coupe (1978) tested the model, they found spatial distortions of this kind, so that San Diego was falsely judged to be west of Reno. 
Further evidence for the role of simple heuristics in spatial representations comes from a study by Tversky (1981). She found a number of spatial memory distortions that she proposed could be accounted for by two storage heuristics: an alignment heuristic and a rotation heuristic. In the alignment heuristic, figures are represented as being more simply aligned than they are in reality. Thus, Tversky found that the pairs North America/Europe and South America/Africa were remembered as being more closely aligned in latitude than is the case. Similarly, North America was recalled as being directly north of South America even though it lies further west than South America. Like Byme (1979), Tversky (1981) also found a memory bias to align intersecting roads at right angles to each other. In the rotation heuristic, Tversky suggests that people misremember the main axis of a figure as being similar to the axis of its frame of reference. For example, Tversky showed that, although the axis of the San Francisco bay peninsula runs from the southeast to the northwest, it is falsely remembered as running south to north. Moar (1979) has shown a similar rotation error, in which the vertical axis of Great Britain is remembered as running south to north rather than in its correct southeast-to-northwest orientation. In both cases, the axis of a figure has been rotated toward the closest main axis of the general frame of reference (e.g., north-south).

The studies of Byrne (1979), Stevens and Coupe (1978), and Tversky (1981) suggest that (1) only partial information is stored about the spatial nature of a known area, (2) storage heuristics are used that simplify the information stored, and (3) spatial information that is not explicitly stored can be inferred from the stored information. These studies bring into question the consistency of spatial information in cognitive maps. For example, a simple storage heuristic may encode a subset of information in a cognitive map without regard to the spatial consistency of that subset with the rest of the cognitive-map representation. Degree of internal consistency here means the extent to which the stored information about an area can be translated into a twodimension euclidean real map. However, to be internally consistent, cognitive maps need not necessarily be represented in a spatial analog format. If a cognitive map is represented in terms of a propositional hierarchy, as suggested by Stevens and Coupe, and if it is internally consistent, the information in the cognitive maps should transfer onto a two-dimensional map. For example, if the location of San Diego in the hierarchy is defined as being in the southwest of California and California is defined as being in the southwest of the United States, such information could be represented in map form. However, if the hierarchy defined Los Angeles as being west of San Diego and San Diego as being west of Los Angeles, this information could not be drawn on a map and so would be inconsistent.

We so far have no evidence that information judged from cognitive maps is spatially inconsistent. For exam- ple, the Stevens and Coupe (1978) finding that San Diego is falsely remembered as being west of Reno does not necessarily mean that cognitive maps of the United States are spatially inconsistent. Reno may also be represented as being east of San Diego, and so the representation would be inaccurate but internally consistent. Similarly, according to Byrne (1979) and Tversky (1981), memory for the angle of turns is biased toward right angles. Again, this does not necessarily imply internally inconsistent cognitive maps. The spatial information in a cognitive map of a town with turns biased toward right angles might still transfer onto a real map. The result would be an inaccurate, simplified, but consistent map. The London underground map is an example of a map containing simplified distance and turn information about the London subway system. Almost all the turns on the map are either $90 \mathrm{deg}$ or $45 \mathrm{deg}$ and yet the map is still consistent. The old New York subway map (New York City Transit Authoity, revised edition, February 1978) is a similar kind of simplified map.

Evidence for the possible inconsistent nature of cognitive maps comes from a study by Holyoak and Mah (1982). Subjects were given a distance scale extending across the United States from the Pacific coast to the Atlantic coast. They then judged how far eastward certain United States cities were from the Pacific coast and how far westward the same cities were from the Atlantic coast. It was found that the cities were judged too far eastward when judging how far eastward they were from the Pacific coast and too far westward when estimating how far westward they were from the Atlantic coast. Holyoak and Mah's results suggest that subjects' eastwest judgments about the cities were spatially inconsistent.

The present two experiments examined the degree of spatial consistency in directional information judged from cognitive maps. The first experiment involved cognitive maps learned by direct experience of an area. Subjects judged from memory the directions between triads of places in a familiar town. Each place in a triad was located at a road intersection at one of the three corners of a three-sided shape formed of street blocks (see Figure 1). If subjects' memory for the angles of the corners of such a shape are biased toward right angles without regard to the spatial consistency of the whole triad, then the three angles formed by subjects' judged directions between the three places in a triad should add up to over $180 \mathrm{deg}$. Such a result would be evidence for spatial inconsistency in subjects' judged directions from their cognitive maps. In the second experiment, cognitive maps acquired from cartographic maps were examined. Subjects judged directions between cities in the United States. For each pair of cities, they judged the direction between the cities in both directions. The direction from one city to another should be the exact inverse of the direction from the second city to the first. If not, this would be further evidence for spatial 


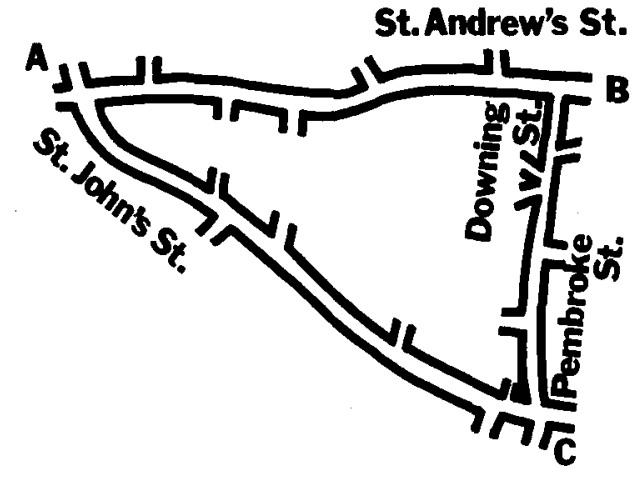

Figure 1. Simplified map of the road triangle formed by one of the triads in Cambridge. The three locations in the triad are the three intersections labeled $A, B$, and $C$.

inconsistency in subjects' judged directions from their cognitive maps.

\section{EXPERIMENT 1}

\section{Method}

Subjects. Subjects were 15 women from the subject panel of the Applied Psychology Unit, Cambridge, England. The women were paid for taking part in the study, and they had all lived in Cambridge for at least 5 years.

Stimuli. Stimuli were nine well known locations situated at road intersections in the center of Cambridge, England. The nine locations formed three triads each with three different locations. An example of a road triangle formed by one of the triads is shown in Figure 1. The number of blocks contained within the road triangle formed by each triad varied from approximately 10 to 40 . The direct "as the crow flies" distance between pairs of locations in the triads varied from 409 to $1,277 \mathrm{~m}$.

Procedure. Subjects were tested individually. Each subject judged 36 directions between triad locations in Cambridge from memory. Half the directions were test directions, and half were fillers used to obscure the purpose of the experiment from the subjects. The 18 test directions comprised the six directions between the three locations in each of the three triads. The 18 fillers comprised two directions from each location in a triad to one of the locations in each of the other two triads.
The experimenter read out the names of the pairs of locations between which directions were judged. For example, a pair of locations were described as "from the junction of St. Andrew's Street and Downing Street facing the direction in which Downing Street leaves the junction to the junction of Pembroke Street and Trumpington Street" (the word "junction" refers to an intersection in British English). This example can be seen in Figure 1.

Subjects were given sheets of paper, each containing a $3.1-\mathrm{cm}$ long arrow in the center pointing toward the top of the sheet with a dot at its base. To judge the direction between a pair of locations, the subject imagined that the dot at the base of the arrow represented the center of the intersection of the first location of the pair and that the direction of the arrow represented the direction at which the specified road leaves that intersection. The subject then drew a straight line from the dot at the base of the arrow in the "as the crow flies" direction of the second location of the pair. A separate sheet was used for each judgment. The order of judgments was randomized differently for each subject.

\section{Results}

For each triad, the three angles were calculated from each subject's judged directions between the three locations in that triad. An angle was calculated from the two judged directions by a subject from a location to the other two locations in the same triad. The same was done for the angles at the other two locations of the triad. Next, one-sample $t$ tests were used to test for biases toward $90 \mathrm{deg}$ in the derived angles. The results are shown in Table 1. All but two of the angles were significantly biased in the direction of $90 \mathrm{deg}$. Of the two angles that were not significantly biased, one was approximately $90 \mathrm{deg}$ in any case (84-deg real angle). In addition, $t$ tests indicated that, across subjects, the sum of the three derived angles for each of the three triads was significantly greater than $180 \mathrm{deg}$ (see Table 1 ). This is strong evidence for spatial inconsistency in subjects' judged directions from their cognitive maps of Cambridge.

One possible reason why the derived angles were biased toward $90 \mathrm{deg}$ is that subjects may have judged directions in terms of the local angles in which the roads

Table 1

Mean Derived Angles and Mean Sum of Derived Angles for Each Triad

\begin{tabular}{|c|c|c|c|c|c|c|c|}
\hline \multirow[b]{2}{*}{ Triad } & \multirow[b]{2}{*}{ Real Angle } & \multicolumn{4}{|c|}{ Derived Angle } & \multicolumn{2}{|c|}{$\begin{array}{l}\text { Sum of Derived Angles } \\
\text { for Each Triad }\end{array}$} \\
\hline & & Mean & SD & $p_{1}$ & $\mathrm{p}_{2}$ & Mean & $\mathrm{p}_{3}$ \\
\hline 1 & $\begin{array}{r}100 \\
53 \\
27\end{array}$ & $\begin{array}{l}88.6 \\
78.1 \\
41.5\end{array}$ & $\begin{array}{l}11.7 \\
22.2 \\
20.3\end{array}$ & $\begin{array}{l}.01 \\
.001 \\
.01\end{array}$ & $\begin{array}{l}\text { n.s. } \\
.05 \\
.001\end{array}$ & 208.1 & .01 \\
\hline 2 & $\begin{array}{l}67 \\
63 \\
50\end{array}$ & $\begin{array}{l}83.9 \\
78.0 \\
87.5\end{array}$ & $\begin{array}{l}19.3 \\
22.6 \\
37.2\end{array}$ & $\begin{array}{l}.01 \\
.05 \\
.01\end{array}$ & $\begin{array}{l}\text { n.s. } \\
.05 \\
\text { n.s. }\end{array}$ & 249.3 & .001 \\
\hline 3 & $\begin{array}{l}84 \\
61 \\
35\end{array}$ & $\begin{array}{l}89.3 \\
90.5 \\
40.1\end{array}$ & $\begin{array}{l}16.5 \\
15.9 \\
25.3\end{array}$ & $\begin{array}{l}\text { n.s. } \\
.001 \\
\text { n.s. }\end{array}$ & $\begin{array}{l}\text { n.s. } \\
\text { n.s. } \\
.001\end{array}$ & 220.0 & .001 \\
\hline
\end{tabular}

Note-Angles are given in degrees. $p$ values are from one-sample $t$ tests: $p_{1}=p$ (derived angles differ from real angles); $p_{2}=p($ derived angles differ from $90 \mathrm{deg}) ; p_{3}=p$ (sum of derived angles differ from $\left.180 \mathrm{deg}\right)$. 
left the intersections. When they imagined they were at one of the intersections, they may have falsely assumed the roads continued from the intersection in straight lines to the other two places in that traid, even though the roads always contained bends. Therefore, they judged the directions to the other two places in the triad in terms of the direction in which the roads initially left the intersection. The local angle of the intersections was closer to $90 \mathrm{deg}$ than the correct angle (formed by direct directions between the three places in a triad) at all but one of the nine triad places used in the study. Thus the overestimation of the derived angles may have been due not to a bias toward 90 deg but to a bias toward the local angle at the intersection.

In order to test whether the derived angles were closer to the local angles or to $90 \mathrm{deg}$, a $t$ test was carried out for each triad place comparing the differences between the derived angles and the local angles with the differences between the derived angles and $90 \mathrm{deg}$. The $t$ test results indicated that the derived angles were significantly more similar to $90 \mathrm{deg}$ than to the local angles at six of the triad places (the local angles varied from 70 to $95 \mathrm{deg}$ ) and were significantly more similar to the local angles than to $90 \mathrm{deg}$ at two of the triad places (the local angles were 32 and $69 \mathrm{deg}$ ). For the one triad place at which the derived angles were not significantly more similar to the local angles or to $90 \mathrm{deg}$, the local angle was $90 \mathrm{deg}$.

Although the $t$-test results initially suggest that subjects biased their derived angles toward $90 \mathrm{deg}$ rather than toward the local angles, it is still possible that subjects judged directions in terms of the local angles. When the local angle was $70 \mathrm{deg}$ or greater, subjects may have remembered it as $90 \mathrm{deg}$ and judged directions in terms of this biased local angle. When the local angle was less than $70 \mathrm{deg}$, they may have expanded the local angle but not to the extent of $90 \mathrm{deg}$ and then used this biased local angle to judge directions. However, as the expansion of the local angle was not as great as $90 \mathrm{deg}$, it may have been possible for the derived angle from these triad places to still be more similar to the local angle than to $90 \mathrm{deg}$.

\section{EXPERIMENT 2}

The results of the first experiment suggest that subjects' judged directions from their cognitive maps of Cambridge were spatially inconsistent. Such cognitive maps are likely to have been acquired largely from direct experience of the area. When learning an area by traveling through it, it is usually not possible to view the entire area from any one location. This is especially the case in a town such as Cambridge, with its narrow twisting streets. In order to learn such an area, the traveler must integrate scenes viewed from different locations. Such a complex process is likely to lead to errors and opens the way for inconsistent spatial encodings.

The second experiment examined the degree of internal consistency in judged directions from well learned cognitive maps that have been acquired from real maps. Subjects judged from memory the directions between pairs of United States cities. For each pair, they judged the direction between the two cities in both directions. If the two judged directions between a pair of cities were consistently not reversible (i.e., one direction of the pair did not equal the other direction minus $180 \mathrm{deg}$ ), this would suggest that such judged information contains contradictory spatial information.

\section{Method}

Subjects. Subjects were 15 undergraduates at the University of Santa Clara, California. They participated as part of their course requirements.

Stimuli and Procedure. Subjects were individually tested. Each subject answered direction questions in five blocks, each containing the same 30 direction questions. A direction question was in the form of the names of two cities. The cities were always chosen from a pool of the same six well known United States cities: Chicago, Miami, New Orleans, New York, San Diego, and Seattle. The direction questions in a block comprised the 30 possible pairwise combinations of the six cities. Thus each pairing occurred twice in a block, once with one city first and once with the other city first. The direction questions were presented in a 30-page response form. Each page contained five different direction questions. The same pair of cities, regardless of order, did not appear more than once on a page. The order in which the direction questions appeared was randomized differently in each form and within each block.

To judge directions, subjects were given a sheet with a circle of $5.0-\mathrm{cm}$ radius drawn in the center of the page. The circumference of the circle was marked with a dash at every $1 \mathrm{deg}$ of arc. These degree markers were numbered every $5 \mathrm{deg}$ from 0 to $355 \mathrm{deg}$. The 0 -deg marker was also labeled as north, the $90 \mathrm{deg}$ marker as east, the 180-deg marker as south, and the 270-deg marker as west. A dot was drawn at the center of the circle. The circle was oriented so that the 0-deg marker (north) was pointing toward the top of the page.

To judge the direction between a pair of cities, subjects imagined that the location of the first city of the pair was represented by the dot in the center of the circle. The direction of north from that city was represented by the direction from the dot to the 0-deg marker labeled north. Subjects then judged the direction to the second city of the pair by estimating the direction from the dot. They wrote the number of the degree marker on the circle circumference that represented their chosen direction on the response form next to the relevant direction question. Subjects answered the direction questions in the order in which they appeared in their response forms.

\section{Results}

Each subject judged the direction between each city pair five times in one direction (e.g., San Diego to Seattle) and five times in the opposite direction (e.g., Seattle to San Diego). For each city pair, the mean of the five directions judged in one direction between a city pair and the mean of the five directions judged in the opposite direction between that city pair were calculated for each subject. The resulting means are called mean judged directions. The standard deviation for the five directions used to calculate each mean judged direction was also obtained. The mean standard deviation across all subjects and city pairs for the five directions judged between a city pair in one direction was $7.8 \mathrm{deg}$.

The degree of nonreversibility in the judged direc- 
tions was calculated as follows. Of the two mean judged directions between each pair of cities (e.g., San Diego to Seattle and Seattle to San Diego) by a subject, $180 \mathrm{deg}$ was added to the smaller of the two directions. Once this transformation was made to one of the mean judged directions, both were then called mean revised judged directions. Next, the difference between the two mean revised judged directions was calculated. This difference is called the unsigned nonreversibility score. The mean unsigned nonreversibility score over all subjects and city pairs was $8.0 \mathrm{deg}$. The mean unsigned nonreversibility scores over all subjects varied from $4.7 \mathrm{deg}$ between San Diego and Seattle to $13.6 \mathrm{deg}$ between New York and Chicago.

The unsigned nonreversibility scores do not indicate whether or not the pattern of nonreversibility in judged directions between a city pair was similar across subjects. For example, a similar nonreversibility pattern would occur if most or all subjects judged Seattle too far west from San Diego and judged San Diego too far west from Seattle. In this case, the judged directions would be nonreversible and the nonreversibility pattern would be similar across subjects. In order to examine the pattern of nonreversibility in the judged directions, the nonreversiblity scores were assigned positive or negative signs in the following manner. For a given city pair, if a subject's mean revised judged direction from the more northern of the two cities was in a more clockwise direction than that subject's mean revised judged direction from the more southern city, the nonreversibility score for that pair of mean revised judged directions was given a positive sign. If the mean revised judged direction from the more northern city was in a more counterclockwise direction than that from the southern city, the nonreversibility score was given a negative sign. The mean signed nonreversibility scores for each city

Table 2

Mean Signed Nonreversibility Scores

\begin{tabular}{|c|c|c|c|}
\hline \multirow[b]{2}{*}{ City Pair } & \multicolumn{2}{|c|}{$\begin{array}{c}\text { Signed Non- } \\
\text { reversibility Score }\end{array}$} & \multirow[b]{2}{*}{$\mathrm{p}^{*}$} \\
\hline & Mean & SD & \\
\hline Seattle/San Diego & 1.4 & 5.7 & n.s. \\
\hline Seattle/New Orleans & -4.2 & 6.7 & .01 \\
\hline Seattle/Miami & -5.3 & 9.2 & .05 \\
\hline Seattle/New York & -5.8 & 11.1 & .05 \\
\hline Seattle/Chicago & -2.5 & 15.5 & n.s. \\
\hline San Diego/New Orleans & 3.7 & 12.3 & n.s. \\
\hline San Diego/Miami & 4.5 & 5.7 & .01 \\
\hline San Diego/New York & 3.2 & 8.9 & n.s. \\
\hline San Diego/Chicago & 5.1 & 10.3 & .05 \\
\hline New Orleans/Miami & -2.2 & 6.8 & n.s. \\
\hline New Orleans/New York & 6.4 & 10.2 & .01 \\
\hline New Orleans/Chicago & 6.7 & 17.3 & n.s. \\
\hline Miami/New York & -3.5 & 5.6 & .01 \\
\hline Miami/Chicago & .5 & 8.2 & n.s. \\
\hline New York/Chicago & -11.6 & 16.2 & .01 \\
\hline
\end{tabular}

pair can be seen in Table 2. If subjects' judged directions were spatially consistent, the expected mean signed nonreversibility score would be $0 \mathrm{deg}$.

For each pair of cities, a t test was used to determine if subjects' signed nonreversibility scores differed significantly from $0 \mathrm{deg}$. The results of the tests are shown in Table 2. For more than half the pairs of cities, the signed nonreversibility scores differed significantly from 0 deg. The results indicate that for more than half the pairs, the judged directions between the cities were consistently not reversible and were not reversible in the same manner across subjects. For example, subjects consistently judged Chicago as being farther north of New York than is the case. However, they also judged New York as being farther north of Chicago than is correct. The evidence strongly supports the hypothesis that subjects' judged directions from their cognitive maps of the United States were spatially inconsistent. The results are analogous to the spatial inconsistencies found in distance estimates for United States cities by Holyoak and Mah (1982).

\section{GENERAL DISCUSSION}

The present research suggests that information judged for cognitive-map representations can be spatially inconsistent in terms of angular and directional properties. Furthermore, inconsistency can occur in judged information from cognitive maps acquired from real maps or by direct experience with an area. These findings were obtained using two different measures of inconsistency: angular distortions derived from judged directions and nonreversibility in judged directions.

The angular distortions found in Experiment 1 indicate large inconsistencies in subjects' judged spatial information from their cognitive maps of Cambridge. The results suggest that the angles of a triad were distorted toward $90 \mathrm{deg}$ without regard to the spatial consistency of the whole triad. The findings support the suggestion of Byrne (1979), Stevens and Coupe (1978), and Tversky (1981) that simple heuristics are used to store or retrieve information in cognitive maps. The results further imply that these simple heuristics can be applied to a subset of information in a cognitive map without regard to the consistency of the spatial information in that subset to the rest of the cognitive map representation.

Evidence for spatial inconsistency in judged information from cognitive maps was also found in Experiment 2 . The judged directions between more than half the pairs of cities were found to be significantly nonreversible. The results suggest that in cognitive maps, the directional relation between two places is stored or retrieved as two distinct pieces of information: the direction from one place to the other (e.g., A to B) and the direction from the other place to the first (e.g., $B$ to A). Although widespread, the magnitude of the nonreversibility effects in Experiment 2 was not large (see Table 2). The relatively small magnitude of the 
scores might reflect a threshold above which subjects could detect the nonreversibility in their judgments and, as a result, change their judgments to make them more spatially consistent.

What distinguished reversible from nonreversible city pairs? One possibility is that local physical features (e.g., coastline) near a city distorted directions judged from that city but directions judged to that city were not distorted by the same features. The physical features around the cities used in the experiment were examined carefully on real maps but could not obviously account for the results. The cause of the nonreversibility effects needs further investigation.

The findings of the present study on the nature of cognitive maps can be accounted for in three different ways. First, cognitive maps contain spatially inconsistent properties. Thus, in Experiment 1, subjects' cognitive maps may have contained inconsistent information about the angles between places in Cambrige. Second, cognitive maps contain consistent spatial information but spatially inconsistent information can be obtained from these representations due to retrieval or inference processes operating on the representations. For example, in Experiment 1, subjects may have had spatially consistent cognitive maps of Cambridge but retrieval or inference processes may have biased derived angles toward $90 \mathrm{deg}$. Third, cognitive maps are composed of subsets of information. Each subset contains spatially consistent properties but spatially inconsistent information can occur between different subsets. In Experiment 1, for example, subjects may have had a spatially consistent subset cognitive map for each of the three places in a triad with angles biased toward $90 \mathrm{deg}$. However, the information contained in any one of the three subset cognitive maps may not have been spatially consistent with the information contained in the other two subset cognitive maps. Similarly, in Experiment 2, subjects may have used one spatially consistent subset cognitive map to judge the direction from one city to another and a different spatially consistent subset cognitive map to judge the opposite direction between the two cities. However, the information in the two subset cognitive maps may have differed, resulting in spatial inconsistency in the form of directional nonreversibility.

The present study has implications for several theories of spatial representation. Piaget and Inhelder (1967) have postulated that children acquire spatial representations in three developmental stages: a topological stage, a projective stage, and, finally, a euclidean stage. Adults are assumed to have spatial representations that contain the functional properties of euclidean space. Similar developmental sequences have been proposed by Hart and Moore (1973) and Siegel and White (1975). In each, adults' spatial representations of well known areas are assumed to have euclidean functional properties. It is also assumed that when adults retrieve or infer information from their euclidean spatial representations, the information obtained conforms to the properties of euclidean space. The present study with adults suggests that even in judgments from cognitive maps of a very familiar town acquired by direct experience, or from cognitive maps acquired from well known real maps, the properties of euclidean space can be violated. Thus the results of the study suggest either that adult cognitive maps are not euclidean in nature or that they are euclidean but do not appear as such in output protocols.

In visual imagery research, the output of spatially inconsistent information from a visual image can be taken as evidence against the analog nature of visual imagery (Kosslyn \& Pomerantz, 1977; Pylyshyn, 1973; Shepard \& Chipman, 1970). Subjects in both the present experiments were asked after testing how they had judged directions. All the subjects in Experiment 1 reported using visual images comprising scenes they would see if they were standing at locations in Cambridge. In Experiment 2, the subjects all claimed they used visual images of cartographic maps of the United States. As subjects in both experiments reported using visual images, do the spatial inconsistency findings of the study argue against the analog nature of visual images? The present findings do not necessarily contradict the analog nature of visual images for two main reasons. First, as was previously discussed, the output of spatially inconsistent information from a spatial representation does not necessarily imply that the representation is spatially inconsistent. The spatial inconsistency may result from retrieval or inference processes operating on a spatially consistent representation. Second, visual imagery research has concentrated on the nature of single visual images. If visual images are used in cognitive maps, information obtained from a cognitive map may involve more than one visual image. Each visual image may contain spatially consistent properties, but spatially inconsistent information may occur between the information contained in the separate visual images. More research is needed on the way we use multiple visual images to retrieve information and make inferences about the world.

In conclusion, the present study suggests the following. (1) Information obtained from cognitive maps can contain inconsistent spatial properties. (2) Spatially inconsistent information from cognitive maps may be due to storage or retrieval processes. (3) Simple heuristics are used to store or retrieve spatial information in cognitive maps, as implied by the bias toward $90 \mathrm{deg}$ in subjects' memory for angles in Experiment 1. (4) In cognitive maps, the direction from one place to another (e.g., A to B) can involve storage or retrieval processes different from those that the reverse direction between the two places (e.g., B to A) involves. As a result, directional information judged from cognitive maps can be nonreversible.

\section{REFERENCES}

BYrNe, R. Memory for urban geography. Quarterly Journal of Experimental Psychology, 1979, 31, 147-154.

Hart, R. A., \& Moone, G. T. The development of spatial cognition: A review. In R. M. Downs \& D. Stea (Eds.), Image and environment. Chicago: Aldine, 1973. 
Hintzman, D. L., O’Dell, C. S., \& ARndt, D. R. Orientation in cognitive maps. Cognitive Psychology, 1981, 13, 149-206.

Holyoax, K. J., \& MAH, W. A. Cognitive reference points in judgments of symbolic magnitude. Cognitive Psychology, 1982, 14, 328-352.

Kosglyn, S. M., \& Pomerantz, J. R. Imagery, propositions, and the form of internal representations. Cognitive Psychology, $1977,9,52-76$.

MoAr, I. Mental triangulation and the internal representation of spatial information. Unpublished doctoral dissertation, University of Cambridge, 1979.

Moar, I., \& Carleton, L. Memory for routes. Quarterly Journal of Experimental Psychology, 1982, 34A, 381-394.

Pinaet, J., Inhelder, B. The child's conception of space. New York: Norton, 1967.

PyLYeryn, Z. W. What the mind's eye tells the mind's brain: A critique of mental imagery. Psychological Bulletin, 1973, 80, $1-24$.
Shepard, R. N., \& Chipman, S. Second-order isomorphism of internal representations: Shapes of states. Cognitive Psychology, 1970, 1, 1-17.

Sizazl, A. W., \& White, S. H. The development of spatial representations of large scale environments. In $\mathbf{H}$. W. Reese (Ed.), Advances in child development and behavior (Vol. 10). New York: Academic Press, 1975.

Stevens, A., \& Coupe, P. Distortions in judged spatial relations. Cognitive Psychology, 1978, 10, 422-437.

TVErsky, B. Distortions in memory for maps. Cognitive Psychology, 1981, 13, 407-433.

WiLTon, $\mathbf{R}$. N. Knowledge of spatial relations: The specification of the information used in making inferences. Quarterly Journal of Experimental Psychology, 1979, 31, 133-146.

(Received for publication September 3, 1982; revision accepted August 24, 1982.) 